\title{
DEVELOPMENT OF AN ANN MODEL TO PREDICT SURFACE ROUGHNESS DURING CRYOGENIC MACHINING OPERATION
}

\author{
K. K. Mandal ${ }^{1}$ \\ ${ }^{1}$ Assistant Professor: Department of Mechanical Engineering, North Eastern Regional Institute of Science and \\ Technology (DU), Nirjuli - 791109, Itanagar, Arunachal Pradesh, India
}

\begin{abstract}
This research paper deals with the advanced manufacturing technique which can be achieved by improving machining parameters through cryogenic cooling approach instead of conventional cooling Industrial growth and globalization aim to increase the material removal rate maintaining very good surface finish and high machining accuracy even for harder, difficult to cut materials. Also, it should be possible at lower overall cost as well as maintaining an eco-friendly environment. Cutting tool fails due to high-elevated temperature and plastic deformation. Also the material is removed due to ploughing and rubbing instead of ideal shearing under conventional cooling method. Moreover, for ductile material cutting, heat causes a "stickiness" which produces material build-up on the cutting edge. This results a bad odor, smoke, health hazards and water penetration into the machine bearings. To improve the surface roughness i.e. to maintain the tool's form stability for longer time, cutting zone temperature is to be maintained low. Liquid nitrogen used as coolant can reduce the cutting zone temperature to a greater extent and thereby helps to retain the shape of cutting edge of cutting tool for a longer time. Also HSTR alloys, MMC may also be machined economically because of low tool wear rate and easy chip breakability. In this present problem, an ANN model has been developed to predict tool wear during cryogenic machining using back propagation feed-forward network algorithm and four hidden layers feed forward architecture.
\end{abstract}

Keywords - Advanced Manufacturing Technique, Conventional Cooling Methods, High Speed Machining, Ideal Shearing, Machining Accuracy, Neural Network Model, Surface Roughness, Cryogenic Cooling. $* * *$

\section{INTRODUCTION}

According to advanced technology, industrial growth and globalization, our aim is to increase the material removal rate i.e. high speed machining, maintaining the consistency of very good surface finish and high machining accuracy. Definition of high speed machining depends on the work-tool combination [1]. Even for harder, difficult to cut materials this target is to be fulfilled at lower overall cost of manufacturing and maintaining eco-friendly environment [1], [2]. Cutting fluid is used to extend tool life by reducing friction between tool, chip and work-piece. However, conventional cutting fluids are environmental pollutants and health hazards as the bacteria and microbial organisms feed on the coolant base oils, rust inhibitors and emulsifiers [2]. Cutting tool fails due to high-elevated temperature and plastic deformation under high speed machining of tough, very hard and difficult-to-cut material using conventional cooling methods. Also material is removed due to ploughing and rubbing instead of ideal shearing as cooling as well as lubrication function of the cutting fluid reduces which inhibits adhesion, rubbing of the chips and welding-like seizure between the chip and the tool [3]. Also for machining ductile materials, long continuous chips can scratch the finished surface [4]. The high elevated temperature increases the temperature dependent wear rapidly which affects on the finished surface. It also causes dimensional deviation and premature failure of cutting tools due to loss of form instability. To improve the surface roughness i.e. to increase tool's form stability, basic conventional approach involves either heating the job or cooling the cutting tool. But these methods do not totally fulfill the above mentioned needs of the high speed metal machining. Also, these techniques are uneconomical as this process needs more idle time [5]. During ductile materials cutting, the generated heat causes a "stickiness" which produces material build-up on the edge of the cutting tool known as "self-welding." It also creates long and continuous chips which often snarl and jam the automatic turning and too bulky for disposal [6]. Also, these types of produced chips may scratch the finished surface [7].

To eliminate above mentioned limitations during advanced metal cutting, cryogenic cooling is getting more importance nowadays. In cryogenic machining using liquid nitrogen $\left(\mathrm{LN}_{2}\right)$ as coolant, is considered a viable option to conventional machining. With temperature as low as $-196.7^{\circ} \mathrm{C}$ at 1 atmosphere, super cold $\mathrm{LN}_{2}$ is a good coolant. It leaves no harmful residue to the environment. So, it is considered naturally recycling and environmentally friendly. Cryogenic machining process improves the tool life up to five times when 
compared with state-of-the-art conventional cooling. This clean process also increases the productivity and lowers the production cost [2]. It helps to remove the material by ideal shearing and less by the ploughing, sliding and rubbing as the chips are irregular, easily removable and thereby reduces the build-up edge [4]. In 1965, Grumman Aircraft Engg. Corpn. reported safe and successful tool-life improvement using very small quantity of $\mathrm{LN}_{2}$ to cool HSS end mills [8]. In different approach, the temperature in the cutting zone can be reduced through cryogenic cooling in turning of stainless steel with diamond tool. It can reduce the cutting zone temperature from $1150^{\circ} \mathrm{C}$ to $830^{\circ} \mathrm{C}$, i.e. $35 \%$ reduction at higher ranges of cutting parameters. With the help of cryogenic cooling, harder materials, HSTR alloys and MMCs may be machined economically mainly because of low tool wear rate as it retain its shape dropping the cutting zone temperature [9]. As tool wear is in the lower range under cryogenic cooling, so, surface finish and job accuracy is also improved a lot comparing to conventional cooling methods [10]. $\mathrm{LN}_{2}$ machining provides lower tool wear, better surface finish and cutting forces. By injecting a small amount of liquid nitrogen to the chip-tool interface yield a noticeable (i.e. $43 \%$ to $67 \%$ ) tool-life as well as surface roughness improvement compared to conventional cooling. It also improved machining productivity and reduced overall production cost [11].

There are so many soft computing methods available to predict the machining performances under different machining parameters. Likewise surface roughness prediction is one of them. Few of them are design of experiment [12], Taguchi Method, Genetic algorithms [13].and so on. Also Artificial Neural Network (ANN) model is used to predict the tool wear [14]). In the present problem, an ANN model has been developed to predict surface roughness during cryogenic machining using back propagation feed-forward network algorithm and single layer feed forward architecture.

\section{EXPERIMENTAL PLANNING}

The experimental work, here turning operation is performed in a PL-4 Lathe of Laxvard Industries, Bangalore. The job material is high strength steel, technically known as hot die steel i.e. $\mathrm{H}_{11}$ is undergone a heat treatment process to achieve the required hardness $\left(R_{C} 58\right)$. To observe the effect of any machining parameter on the finished surface, tool is changed at the end of every cutting. For this purpose, replaceable tool inserts are used. The specification of tungsten carbide insert is CC MT 090304 TK 15/TN 200. Shape of these tool inserts is rhomboidal with an angle of $80^{\circ}$. Experiments are performed under the cryogenic environment. Before starting the turning operation, job is slowly rotated and liquid nitrogen spray is applied over the job through a movable nozzle to lower down the ductility of workpiece. This process is known as precooling the job, tends to improvement of chip breaking and thereby avoiding scratching over the finished surface. Details of the experimental conditions selected to fulfill the criteria are given in Table 1. After each set of experiments, surface roughness is measured by Handy Surf.

Table1: Experimental Conditions

\begin{tabular}{|l|l|}
\hline ITEMS & DESCRIPTION \\
\hline Cutting Speed & $45,50,55 \mathrm{~m} / \mathrm{min}$ \\
\hline Depth of Cut & $0.5,0.75,1.0,1.5 \mathrm{~mm}$ \\
\hline Feed Rate & Constant at $1 \mathrm{~mm} / \mathrm{rev}$ \\
\hline Length of Cut & $25,50,75,100 \mathrm{~mm}$ \\
\hline Equipment Used & Handy surf \\
\hline Environment & Liquid Nitrogen Flow \\
\hline
\end{tabular}

\section{CRYOGENIC MACHINING}

With the advanced machining technology i.e. at higher MRR, heat build-up is greater and cutting temperature is so high that cooling effect by conventional cooling method is no longer effective to reduce temperatures. The method applied and apparatus used for the present invention provide a practical solution for the improved machining of materials including HSS alloys, titanium, ceramics and composites. This invention has special advantage in improving chip breaking for ductile material which may be obtained due to following mechanisms: a) Increasing the brittleness of the material.

b) Reducing the tendency of secondary deformation of chip which rubs on tool face.

c) Reducing long tail in the grain structure of the chips which helps in breaking chips.

d) Avoiding self-welding of the bottom layer of the chip due to coldness.

e) Bending and curling the chip for breaking due to the pressure from the cryogenic fluid.

In this invention, build-up edge formation is automatically prevented by injecting the super cold cryogenic fluid to the cutting zone. This reduces the adhesion of chip to the tool tip which lowers down the temperature. Also, it can clean possible build-up edge with the cryogenic jet due to which the present invention also indirectly improves the finish of the machined surface. This provides a fluid cushion which reduces the friction of the chip on the tool face, and reduces the cutting force involved, as well as the abrasive wear. Thus friction between the chip and the tool face is greatly reduced and therefore, heat due to the frictional deformation is also reduced.

\section{DEVELOPMENT OF THE NEURAL NETWORK MODEL}

Generally, an ANN is made of an input layer of neurons, one or several hidden layer(s) of neurons and an output layer of neurons. These neurons are sometimes referred to as nodes or processing units. The neighboring layers are fully interconnected by different weights decided by the different transfer functions or weightage like 'logsig', 'tansig', 'purelin', 
etc. The input layer neurons receive information from the outside environment (i.e. input parameters. Here, cutting speed, depth of cut and length of cut) and transmit them to the neurons of the hidden layers without performing any calculation. The hidden layer neurons then process the incoming information and extract useful features to reconstruct the mapping from the input space to output space (i.e. output parameters. Here, surface roughness). Procedure for developing the optimized model consists of two phases namely training phase and testing phase. In this method, backpropagation network is used to learn the forward relationship between the process inputs and process output. Actually the total development procedure for the proposed ANN model includes following steps:

Step 1: Learning rate, Momentum rate, Maximum allowed system error and number of iterations to optimize the model are set.

Step 2: Normalize all the inputs and output variables in the range of 0.0 to 1.0 .

Step 3: Initialize the number of hidden layers and that of neurons in each layer. Also set the model input and output layers.

Step 4: Initiate the connecting weights in normalized scale, bias values and coefficients of the transfer functions in random order.

Step 5: Update connecting weights, bias values and coefficient of transfer functions iteratively using a back propagation algorithm through batch mode training.

Step 6: Set a goal during training process so that the iterations continuously go on until the termination criterion or goal is reached. Also the maximum numbers of epochs or cycles are mentioned to execute from the model during training process. When the model is properly trained then model output is noted and compared with actual output.

Step 7: After getting trained and optimized neural network model, it is tested with non-familiar sets of input values to get the output response and compare with the real value.

Step 8: Number of neurons in hidden layers and the transfer functions for each layer, are taken with different combinations of transfer functions as well as the number of neurons in each hidden layers for optimizing the model.

The Matlab program consists of two parts, one part can be used for continuous training of the model until the goal is reached and when the model gets optimized, having least MSE (mean square error) then the other part of the program i.e. testing is used to compare the surface roughness for unknown sets of input parameters. From the verification data a comparative study revealed that the mean difference between ANN output and experimental output is least when the transfer function between the input layer, hidden layers and output layer are 'logsig', 'tansig', 'logsig' and 'purelin' respectively. Also, here the most suitable number of neurons in hidden layers is found to be $20,20,20,1$.
Finally a feed forward artificial neural network of type $3-20$ $-20-20-1-1$ was adopted to model the process as shown in Figure 1. This is then then used for training as well as testing the machining data.

\section{RESULTS AND DISCUSSION}

The verification data set for optimized model for the training is illustrated in Figure 2. All verification data set for testing is also plotted as shown in Figure 3.

Figure 2 represents comparison between actual experimental outputs versus model output result for surface roughness when the model well trained. For the ideal case all points should lie on line drawn $45^{\circ}$ to the both axis. On the other way it can be said that if all points are joined then a firm solid line is seen. It is observed that there is very small deviation between actual experimental outputs and model output result which is represented by the wide line.

Figure 3 shows slight deviation between actual experimental outputs and model output result for surface roughness when the neural network is tested for a set of unknown output data after the training. These data are collected through experimentation, so range of error by the model is acceptable.

\section{CONCLUSIONS}

The study conducted in this paper shows the feasibility of using a simple ANN model to predict the surface roughness as the output parameter during cryogenic machining from a given sets of input parameters like cutting speed, depth of cut and length of cut. I have used linear output transfer function (purelin) in between hidden layer \& output layer and sigmoid transfer function in between other layers like logsig - tansig logsig which helps in fast converging, to reach the goal. The results indicate that with proper training, ANN can provide an alternative method for predicting surface roughness like other performances during cryogenic machining in cases where it is difficult to model the complex interaction among the multiple variable. The optimized model developed for training data provides very good result for testing data also. Extrapolation over those limits would restrict applicability of the model.

\section{REFERENCES}

[1]. H. Schulz, "Aspects in Cutting Mechanism in High Speed Cutting", Annals of the CIRP, pp. 179-188, Vol. 38, No. 1, January 1989.

[2]. Shane Y. Hong, "Economical and Ecological Cryogenic machining", J. of manufacturing Scienceand Engineering, ASME, pp. 331 - 338, Vol.123, May 2001.

[3]. A. Bhattacharya, "Metal Cutting Theory and Practice", Central Book Publishers (P) Ltd., New Delhi, pp. 117-186 \& 376-415, 2000.

[4]. S. Y. Hong, Y. Ding, R. G. Ekkens, "Improving Low Carbon Steel Chip Breakability by Cryogenic Chip Cooling”, 
Int. J. Machine Tools and Manufacture, pp. 1065 - 1085, Vol. 39, July 1990.

[5]. C. Evans, "Cryogenic Diamond Turing of Stainless Steel", Annals of the CIRP, ), pp. 571-575, Vol. 40, No. 1, January 1991.

[6]. Y. Ding, S. Y. Hong, "Improvement of Chip Breaking in Machining Low Carbon Steel by Cryogenically Precooling the Workpiece”, Trans. ASME, pp. 76-83, Vol.120, No. D, 1998.

[7]. S. Paul, A. B. Chattopadhyay, "The Effect of cryogenic Cooling on Grinding Forces", Int. J. Machine Tools \& Manufacture, pp. 63-72, vol. 36, January 1996.

[8]. S. Ravi, M. P. Kumar, "Experimental investigations on cryogenic cooling by liquid nitrogen in the end milling of hardened steel", Cryogenics, pp. 509-515, Volume 51, 2011.

[9]. Z. Y. wang, K. P. Rajurkar, M. Murugappan, "Cryogenic PCBN Turing of Ceramics (Si3 N4 )", Wear, pp. 1 -6, Vol. 195, 1996.

[10]. K. Uehara, S. Kumagai, "Surface Roughness and Cutting Forces in Cryogenic Machining”, Annals of CIRP, pp. 409, Vol.17, 1969.
[11]. K. K. Mandal, S. Mitra, B.Bhattacharya, "Applying Liquid Nitrogen as the Coolant: An Index for Improving manufacturability", National Conf. on Design for Product Life Cycle, BITS, Pilani, Rajastan, February 2006.

[12]. I. A. Choudhury, M. A. El-Baradie, "Surface roughness prediction in the turning of high-strength steel by factoral design of experiments", Material Processing Technology, pp. 55-61, Vol. 67, 1997.

[13]. P. V. S. Suresh, P. Ventateswara Rao, "Selection of Optimal Machining Conditions for required surface finish using genetic algorithyms", Proc. Of 19th AIMTDR, Narosa Publishing House, New Delhi, pp. 439 - 444, 2000.

[14]. R. Banik, R. Pongen, A. Mandal and K. K. Mandal, "Development of an ANN Model to Predict Tool Wear During Cryogenic Machining", Int. Conf. on Advances in Materials and Manufacturing Technology, Mech. Engg. Deptt. , Chitkara University, pp. $28-30$, July, 2011.

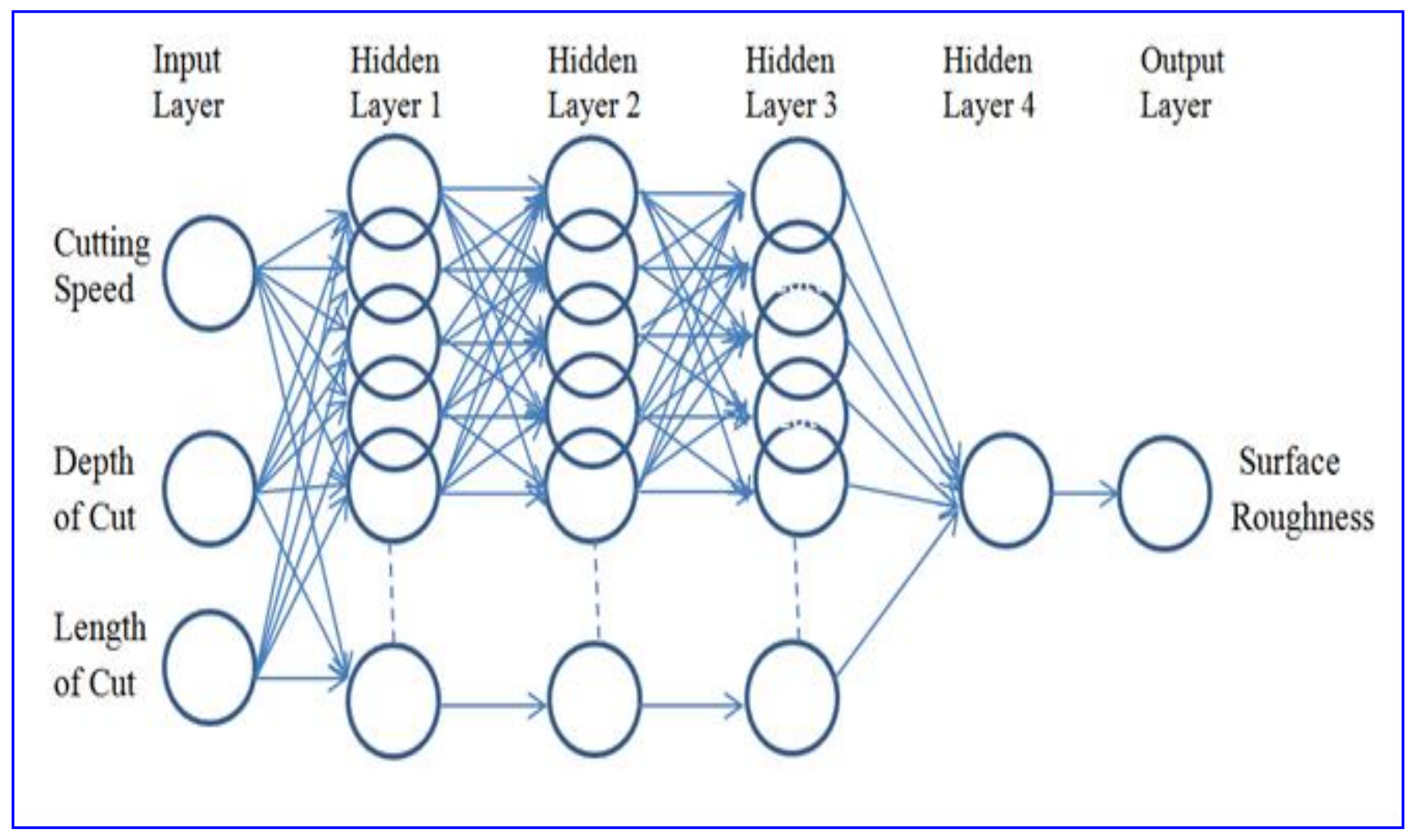

Fig 1 Configuration of Artificial Neural Network 


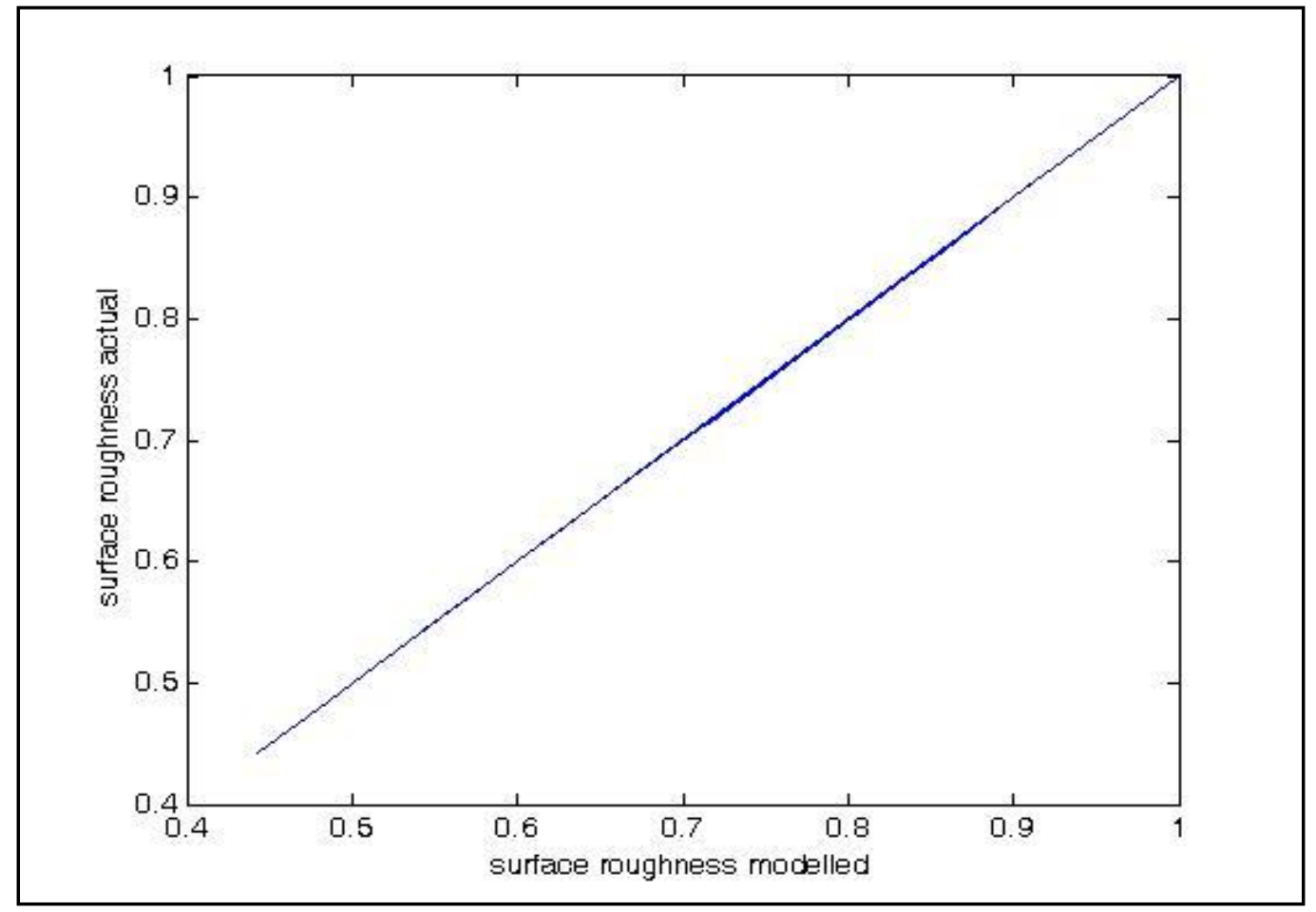

Fig 2 Experimental outputs V/s model outputs for surface roughness for training

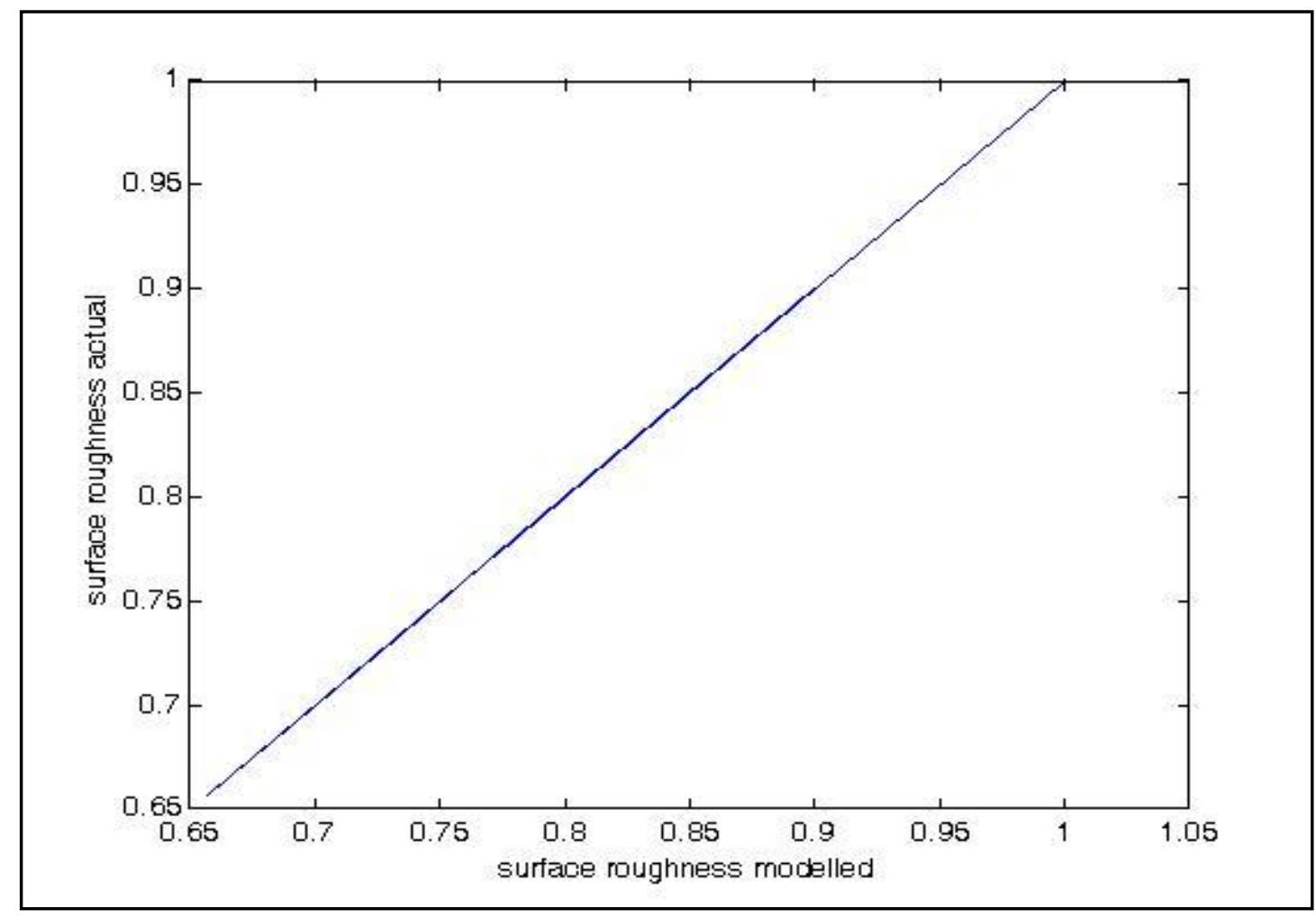

Fig 3 Experimental outputs V/s model outputs for surface roughness for testing 